\title{
MAV/UAV task coalition phased-formation method
}

\author{
JIAO Zhiqiang*, YAO Peiyang, ZHANG Jieyong, ZHONG Yun, and WANG Xun \\ Information and Navigation College, Air Force Engineering University, Xi' an 710077, China
}

\begin{abstract}
The formation of the manned aerial vehicle/unmanned aerial vehicle (MAV/UAV) task coalition is considered. To reduce the scale of the problem, the formation progress is divided into three phases. For the task clustering phase, the geographical position of tasks is taken into consideration and a cluster method is proposed. For the UAV allocation phase, the UAV requirement for both constrained and unconstrained resources is introduced, and a multi-objective optimal algorithm is proposed to solve the allocation problem. For the MAV allocation phase, the optimal model is firstly constructed and it is decomposed according to the ideal of greed to reduce the time complexity of the algorithm. Based on the above phases, the MAV/UAV task coalition formation method is proposed and the effectiveness and practicability are demonstrated by simulation examples.
\end{abstract}

Keywords: task coalition formation, task clustering, unmanned aerial vehicle (UAV) allocation, manned aerial vehicle (MAV) allocation.

DOI: $10.21629 / J S E E .2019 .02 .18$

\section{Introduction}

With the rapid development of science and technology, the combat capability of the unmanned aerial vehicle (UAV) continues to improve. In the future battlefield, the UAV will take the place of the manned aerial vehicle (MAV) to execute tasks [1]. However, due to the complex and changeful battlefield environment, it is unfeasible for the UAV to accomplish the variegated tasks without the MAV at the present stage. As a result, the task coalition which is composed of the MAV and UAV can perform much better on the battlefield, and it will be a main research direction in the future for a long time [2,3]. In the task coalition, the MAV, which has a strong situation awareness and short decision cycle, acts as the role of the commander. The UAV, which has a low production cost and strong endurance, plays the role of the executor. In this way, the command

Manuscript received November 28, 2017.

*Corresponding author.

This work was supported by the National Natural Science Foundation of China (61573017; 61703425), and the Aeronautical Science Fund (20175796014). and decision ability of the pilot and the advantage of the UAV to complete high risk tasks are combined to improve the operational capability. Therefore, the problem of how the MAV/UAV executing the combat tasks together has attracted extensive attention of many research institutions, and a large amount of research has been done in decision making [4], permission assignment [5] and task allocation [6-8].

As the important concept of the multi-agent, coalition formation is the basis for the UAV to accomplish tasks cooperatively. With the increasing complexity of combat tasks, selecting multiple suitable UAVs to form task allocation according to the position, type and resource requirement of tasks has become a development trend. In [9], inter-task resource constraints in task allocation was considered. In this approach, not only the utility of the assignment but also the expected loss of utility (due to the assigned robots and task) are taken into consideration. In [10], the task coalition formation was divided into three phases to reduce the scale of problem solving. The quality of the formation scheme may decrease to a certain extent, but this strategy has advantages in timeliness. In [11], the problem of the decentralized task assignment was considered. To maximize the information investigated by UAVs, the UAV arrival time index and the UAV-task time index are designed to measure the UAV fight distance and linear distance from UAVs to task-areas. An extension of the consensus-based bundle algorithm is proposed to achieve the conflict free solution in an acceptable time.

With the increasing number of the task, in some resource-critical situation, the resource redundancy of the UAVs should be taken into consideration. In [12], the coalition formation method was designed for the task allocation in both multi-agent and multi-robot domains based on the greedy algorithm and achieved the exactly or approximated result within the polynomial time. In [13], the MAV/UAV task allocation problem was analyzed based on the Holon coalition formation theory, and the cluster resource redundancy index and the resource redundancy 
variance index were defined to help establish the multiobjective optimization model. In [14], the task coalition concept was introduced into the MAV/UAV task allocation process and referred to the ideas of the phased formation. The method divides the task coalition formation into two phases: the task clustering phase and the MAVUAV-task cluster phase, which achieves a better result in the formation process. However, the differences between the requirement of tasks and UAVs are not taken into consideration and it also makes the calculation much difficult once putting the match process of the UAV-task cluster and MAV-UAV into one phase.

In summary, the core of the MAV/UAV task coalition formation is that, given a set of MAVs, UAVs and tasks, how to achieve the coalition formations (consist of MAVs and UAVs) that all the tasks can be executed successfully. The research mentioned above can provide certain enlightenment for the MAV/UAV task coalition formation and there still remain some problems that deserve to be paid attention to: (i) When executing the tasks, some resources can be used for many times and the other can only be used for one time, thus the resources of UAVs and MAVs can be modeled in a more reasonable way. (ii) The utility and resource redundancy can be considered at the same time to make the task coalition formation more realistic. (iii) When the numbers of tasks, UAVs and MAVs increase, the scale of the entire problem is too large to achieve the solution in an acceptable time.

In order to describe the task requirements and MAV/UAVs abilities more reasonable and reduce the scale of the entire problem, a phased-formation method for MAV/UAV task coalition is proposed. The innovation and main contributions in this work are as follows:

(i) To reduce the scale of the MAV/UAV task coalition formation problem, this paper divides the formation process into three phases: the task clustering phase, the UAV allocation phase and the MAV allocation phase. Then the three optimization models are established according to the characteristics of each phase respectively.

(ii) In the task clustering phase, this paper takes the intra cluster task distance as an optimize objective to reduce the distance between each task in the same cluster. In this way, the MAVs and UAVs can save the resource and time when executing the tasks in a task cluster. Considering the selection of initial tasks and the mutual distance between the tasks in the cluster, a greedy clustering algorithm based on the task distance is proposed to solve the problem in the task clustering phase, which can achieve a better performance within an acceptable time.

(iii) In the UAV allocation phase, to describe the UAV ability and task requirement in a more subtle way, both constraint and unconstraint resources are taken into consideration at the same time. The multi-objective optimization problem is established to maximize the utility and reduce the resource redundancy. Finally, a multi-objective genetic algorithm based on the constraint violation degree is proposed to solve the problem in the UAV allocation phase, in which the constraint violation degree function is designed to ensure that the constraints can be satisfied by each individual. The performance of the algorithm is superior to the multiple objective particles swarm optimization algorithm and NSGA-II with the classical penalty function.

(iv) In the MAV allocation phase, an optimization model is constructed by reducing the command and control resource redundancy. To reduce the time expenses of the MAV allocation phase, this paper decomposes the optimization problem into multiple sub-optimization problems which are easy to be solved and an MAV allocation algorithm with decomposition is proposed. Compared with the genetic algorithm and the global branch and bound algorithm, it can achieve the approximate global optimal solution without enormous time expenses.

\section{Problem statement}

To help understand the MAV/UAV task coalition formation process, the following concepts should be defined:

(i) Task cluster. The task cluster is a set of tasks, which is formed according to a rule, in which the tasks have some similar characteristics. The task cluster is divided based on the geographical location. It means that the tasks in the same task cluster have a similar geographic location.

(ii) Resources. Resources are the basic units that cannot be separated during the operation. Different types of resources have different capabilities. Usually, tasks require a certain amount of resources for execution, and the platform (UAV or MAV) also has one or more resources. Resources are divided into two types. One is the constraint resource, which is indispensable during the process of task execution. For example, in a reconnaissance operation, the UAV is required to have the ability of infrared detection. If a type of UAVs is not equipped with an infrared detector, the UAV cannot fulfill its task. The other is the unconstraint resource, that is, the number of this resource will affect the completion quality of the task. For example, in a bombing operation, the UAV is needed to launch four bombs, and if the UAV has two bombs, the quality of the task completion would be affected.

(iii) Resource costs. Resource costs represent the redundancy of UAVs' constraint resources when executing the task. If the UAVs have more resources than the requirement of the task, a part of resources will be wasted. The greater redundancy means the greater waste of resources. 
(iv) Resource satisfaction degree. It reflects the satisfaction degree of unconstraint resources. If the UAVs' unconstraint resources do not meet the needs of tasks, the quality of the execution will be affected.

The key problem of the MAV/UAV task coalition formation is to decide which MAVs command which UAVs to execute which tasks. In this paper, the MAV/UAV task coalition formation problem is divided into three phases as shown in Fig. 1. The first phase is the task clustering phase. The main problem is to achieve a classification scheme so that tasks with the similar geographical position can be clustered in the same task cluster. In this way, the resource consumption of the task coalition can be reduced. The second phase is the UAV allocation phase. The core of the phase is to allocate the execution platform (UAV) to the task clusters so that the cost of constraint resources can be minimized and the resource satisfaction degree can be maximized. The third phase is the MAV allocation phase. The main problem to be solved is how to assign the command platform (MAV) to the UAVs which are formed in the second phase to minimize the cost of MAVs' command and decision resources.

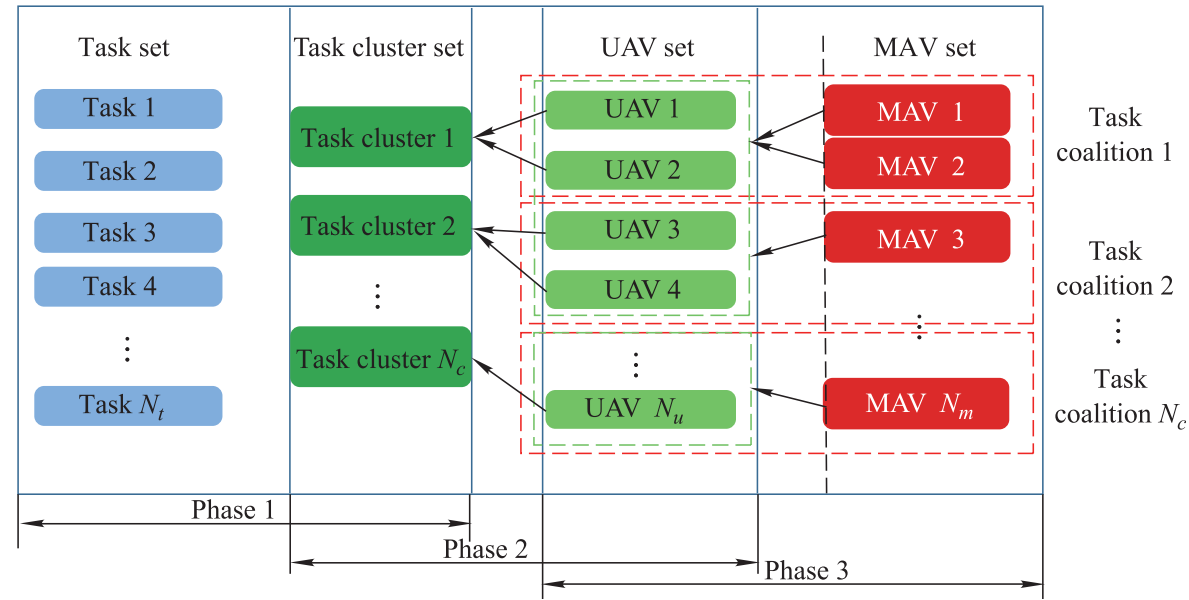

Fig. 1 Three phases of MAV/UAV task coalition formation

\subsection{Task clustering phase}

When the task coalition executes tasks in the task clustering, it needs to arrive at the position of tasks in turn. The distance between tasks can be defined as

$$
D\left(T_{i}, T_{j}\right)=\left\|\boldsymbol{P} \boldsymbol{T}_{i}-\boldsymbol{P} \boldsymbol{T}_{j}\right\|
$$

where $\boldsymbol{P} \boldsymbol{T}_{i} \in \mathbf{R}^{n_{p}}$ and $\boldsymbol{P} \boldsymbol{T}_{j} \in \mathbf{R}^{n_{p}}$ denote the positions of task $i$ and task $j . \mathbf{R}^{n}$ is the $n$-dimensional Euclidean space.

Since the execution order of tasks within a cluster cannot be determined at this phase, it is reasonable to minimize the distance between all the tasks in the task cluster to reduce the resource consumption of the task coalition. For the $i$ th task cluster $T c_{i}$, the definition of its intra cluster task distance is shown as follows:

$$
D T c_{i}=\frac{1}{2} \sum_{j=1}^{N_{T}} \sum_{k=1}^{N_{T}}[\boldsymbol{x}]_{i, j}[\boldsymbol{x}]_{i, k} D\left(T_{j}, T_{k}\right)
$$

where $N_{T}$ denotes the number of tasks, $[\boldsymbol{x}]_{i, j}$ is the element at the $i$ th row and $j$ th column in the task clustering matrix $\boldsymbol{x} \in \mathbf{R}^{N_{T c} \times N_{T}}$. If the task $j$ belongs to the task cluster $i,[\boldsymbol{x}]_{i, j}=1$; otherwise, $[\boldsymbol{x}]_{i, j}=0$. Considering that the number of tasks in each task cluster should be evenly distributed during actual combat operations, the number of tasks in each task cluster should satisfy the constraint

$$
N_{T c_{i}}=\sum_{j=1}^{N_{T}}[\boldsymbol{x}]_{i, j} \leqslant\left\lceil\frac{N_{T}}{N_{T c}}\right\rceil, \quad i=1,2, \ldots, N_{T c}
$$

where $N_{T c}$ denotes the number of task clusters. To ensure every task cluster can achieve the minimum intra cluster task distance, the optimization problem can be defined as follows.

Problem 1

$$
\begin{gathered}
\min _{\boldsymbol{x}} \frac{1}{2 N_{T c}} \sum_{i=1}^{N_{T c}} \sum_{j=1}^{N_{T}} \sum_{k=1}^{N_{T}}[\boldsymbol{x}]_{i, j}[\boldsymbol{x}]_{i, k} D\left(T_{j}, T_{k}\right) \\
\text { s.t. }\left\{\begin{array}{l}
{[\boldsymbol{x}]_{i, j}=0 \text { or } 1, \quad i, j=1,2, \ldots, N_{T}} \\
\sum_{j=1}^{N_{T}}[\boldsymbol{x}]_{i, j} \leqslant\left\lceil\frac{N_{T}}{N_{T c}}\right], \quad i=1,2, \ldots, N_{T c}
\end{array}\right.
\end{gathered}
$$

Compared with the task clustering model [14], the proposed model can describe the mutual distance of tasks more meticulously, so as to reduce the resource consumption. 


\subsection{UAV allocation phase}

The execution of the task requires the UAVs to provide resources. Most of the previous research focuses on the resource satisfaction degree of the allocation, that is, the resources provided by the UAVs should try its best to meet the requirements of the tasks. Resources can be divided into two categories, one is constrained resources, and the other one is unconstrained resources. For unconstrained resources (the number of bombs carried by a fighter, etc.), using the resource satisfaction degree to describe the quality of the task execution is acceptable. However, the lack of constrained resources (such as airborne radar detection capabilities, etc.) will lead to the failure of the task execution directly, thus simply using the resource satisfaction degree to describe the quality of the task execution is not reasonable. The minimum redundancy is taken as the target when assigning UAVs, and the task execution needs UAVs to meet all resource requirements [14]. Obviously, this allocation method can guarantee the quality of the task execution, but it demands that the resources provided by the UAVs are abundant enough. However, resources will not always be sufficient in practice. Therefore, this paper gives full consideration to the influence of two kinds of resources on the task execution quality, and designs the UAV allocation method, including the following two optimization objectives.

(i) Reduce the redundancy of constrained resources under the situation that all the constrained resources are satisfied. The redundancy of constrained resources can be defined as

$$
\begin{gathered}
R D T=\sum_{i=1}^{N_{L}} \sum_{j=1}^{N_{T c}}[\boldsymbol{R} \boldsymbol{d} \boldsymbol{t}]_{i, j} \\
\boldsymbol{R d} \boldsymbol{t}=\boldsymbol{A} \boldsymbol{l}^{\mathrm{T}} \boldsymbol{y}-\boldsymbol{R} \boldsymbol{l}
\end{gathered}
$$

where $\boldsymbol{R} \boldsymbol{d} \boldsymbol{t} \in \mathbf{R}^{N_{L} \times N_{T c}}$ denotes the redundancy matrix of the constrained resource, $N_{L}$ represents the number of constrained resources, $\boldsymbol{A l} \in \mathbf{R}^{N_{\mathrm{UAV}} \times N_{L}}$ denotes the constrained resources matrix which UAVs can provide, $N_{\mathrm{UAV}}$ represents the number of UAVs. $\boldsymbol{y} \in \mathbf{R}^{N_{\mathrm{UAV}} \times N_{T C}}$ is the UAV allocation matrix. If $j$ is allocated to the task cluster $k,[\boldsymbol{y}]_{j, k}=1$; otherwise, $[\boldsymbol{y}]_{j, k}=0 . \boldsymbol{R} \boldsymbol{l} \in \mathbf{R}^{N_{L} \times N_{T c}}$ denotes the constrained resources requirement matrix.

(ii) Maximize the unconstrained resource satisfaction degree. The unconstrained resource satisfaction degree can be defined as

$$
A C C=\left(\prod_{i=1}^{N_{U}} \prod_{j=1}^{N_{T c}}[\boldsymbol{S} \boldsymbol{d}]_{i, j}\right)^{\frac{1}{N_{U} N_{T c}}}
$$

$$
[\boldsymbol{S d}]_{i, j}=\left\{\begin{array}{l}
\min \left(1,\left[\boldsymbol{A} \boldsymbol{n}^{T} \boldsymbol{y} \cdot / \boldsymbol{R u}\right]_{i, j}\right), \quad[\boldsymbol{R u}]_{i, j}>0 \\
1, \quad[\boldsymbol{R u}]_{i, j}=0
\end{array}\right.
$$

where $\boldsymbol{S d} \in \mathbf{R}^{N_{U} \times N_{T c}}$ denotes the unconstrained resource satisfaction degree matrix, $N_{U}$ represents the number of unconstrained resources, $\boldsymbol{A} \boldsymbol{u} \in \mathbf{R}^{N_{\mathrm{UAV}} \times N_{U}}$ denotes the unconstrained resources matrix which UAVs can provide, $\boldsymbol{R} \boldsymbol{u} \in \mathbf{R}^{N_{U} \times N_{T c}}$ denotes the unconstrained resources requirement matrix.

During the UAV allocation process, these constraints should be obeyed:

(i) To ensure the tasks can be executed successfully, all the constrained resource requirements should be satisfied:

$$
\begin{gathered}
{[\boldsymbol{R} \boldsymbol{d} \boldsymbol{t}]_{i, j} \geqslant 0,} \\
i=1,2, \ldots, N_{L} ; j=1,2, \ldots, N_{T c} .
\end{gathered}
$$

Every element in the redundancy matrix of constrained resources must be greater or equal to zero.

(ii) To ensure the quality of the task execution, every element in the unconstrained resource satisfaction degree matrix must be greater or equal to $\delta_{U}$ :

$$
\begin{gathered}
{[\boldsymbol{S} \boldsymbol{d}]_{i, j} \geqslant \delta_{U},} \\
i=1,2, \ldots, N_{U} ; j=1,2, \ldots, N_{T c}
\end{gathered}
$$

where $\delta_{U}$ denotes the minimum satisfaction degree for one resource.

(iii) A UAV can only be assigned to one task cluster at most:

$$
\sum_{j=1}^{N_{T c}}[\boldsymbol{y}]_{i, j} \leqslant 1, \quad i=1,2, \ldots, N_{\mathrm{UAV}} .
$$

In summary, the optimization problem of UAV allocation can be described as follows.

\section{Problem 2}

$$
\begin{aligned}
& \qquad\left\{\begin{array}{l}
\min R D T \\
\max A C C
\end{array}\right. \\
& \text { s.t. }\left\{\begin{array}{l}
{[\boldsymbol{R} \boldsymbol{d} \boldsymbol{t}]_{i, j} \geqslant 0, \quad i=1,2, \ldots, N_{L} ; j=1,2, \ldots, N_{T c}} \\
{[\boldsymbol{S} \boldsymbol{d}]_{i, j} \geqslant \delta_{U}, \quad i=1,2, \ldots, N_{U} ; j=1,2, \ldots, N_{T c}} \\
\sum_{T=1}^{N_{T c}}[\boldsymbol{y}]_{i, j} \leqslant 1, \quad i=1,2, \ldots, N_{\mathrm{UAV}} \\
j=1 \\
{[\boldsymbol{y}]_{i, j}=0 \text { or } 1, \quad i=1,2, \ldots, N_{\mathrm{UAV}} ;} \\
j=1,2, \ldots, N_{T c}
\end{array}\right.
\end{aligned}
$$

\subsection{MAV allocation phase}

In an MAV/UAV task coalition, MAV usually acts as the commander to complete the command and control process. Considering the different command and control ability demands of different UAVs, it is reasonable to allocate the 
MAV on the basis of the UAV allocation scheme, which is achieved in Section 2.2, to satisfy the ability requirement of the UAVs in each group. Drawing on the concept of the resource redundancy, this section takes the command and control resource redundancy into consideration. The redundancy of the command and control resource can be defined as

$$
\begin{gathered}
C C R=\sum_{i=1}^{N_{C}} \sum_{j=1}^{N_{T c}}[\boldsymbol{C} \boldsymbol{c r} \boldsymbol{r}]_{i, j} \\
\boldsymbol{C} \boldsymbol{c r} \boldsymbol{r}=\boldsymbol{C} \boldsymbol{a}^{\mathrm{T}} \boldsymbol{z}-\boldsymbol{C r}
\end{gathered}
$$

where $\boldsymbol{C} \boldsymbol{c r} \boldsymbol{r} \in \mathbf{R}^{N_{C} \times N_{T c}}$ denotes the command and control resource redundancy matrix, $N_{C}$ represents the number of command and control resource, $N_{\mathrm{MAV}}$ represents the number of MAVs. $\boldsymbol{z} \in \mathbf{R}^{N_{\mathrm{MAV}} \times N_{T c}}$ denotes the MAV allocation matrix. If $j$ is allocated to the task cluster $k$, $[\boldsymbol{z}]_{j, k}=1$; otherwise, $[\boldsymbol{z}]_{j, k}=0 . \boldsymbol{C r} \in \mathbf{R}^{N_{C} \times N_{T c}}$ denotes the command and control resource requirement matrix.

In order to ensure the successful implementation of tasks in the task cluster, the requirements for command and control resource must be satisfied:

$$
\begin{gathered}
{[\boldsymbol{C} \boldsymbol{c r} \boldsymbol{r}]_{i, j} \geqslant 0,} \\
i=1,2, \ldots, N_{U} ; j=1,2, \ldots, N_{T c} .
\end{gathered}
$$

The optimization problem of the MAV allocation can be described as follows.

\section{Problem 3}

$$
\begin{gathered}
\min _{\boldsymbol{z}} C C R \\
\text { s.t. } \begin{cases}{[\boldsymbol{C c} \boldsymbol{r} \boldsymbol{r}]_{i, j} \geqslant 0, \quad i=1,2, \ldots, N_{U} ; j=1,2, \ldots, N_{T c}} \\
\sum_{j=1}^{N_{T c}}[z]_{i, j} \leqslant 1, \quad i=1,2, \ldots, N_{\mathrm{MAV}} \\
{[z]_{i, j}=0 \text { or } 1, \quad j=1,2, \ldots, N_{\mathrm{UAV}} ; j=1,2, \ldots, N_{T c}}\end{cases}
\end{gathered}
$$

\section{Task coalition formation method}

\subsection{Task clustering algorithm}

Task clustering is based on the geographical location of the task, which is essentially a clustering problem. For this problem, the existing algorithm, such as GCWOICC [14] $k$-means [15] and so on, can give a reasonable result. However, all the above algorithms aim at minimizing the distance between each point and the center of the cluster, without considering the mutual distance between the points in the cluster. Therefore, the distance between points in the cluster is taken into consideration and a greedy clustering algorithm based on task distance (GCABTD) to solve Problem 1 is proposed.

The flowchart of the GCABTD algorithm is shown in Fig. 2.

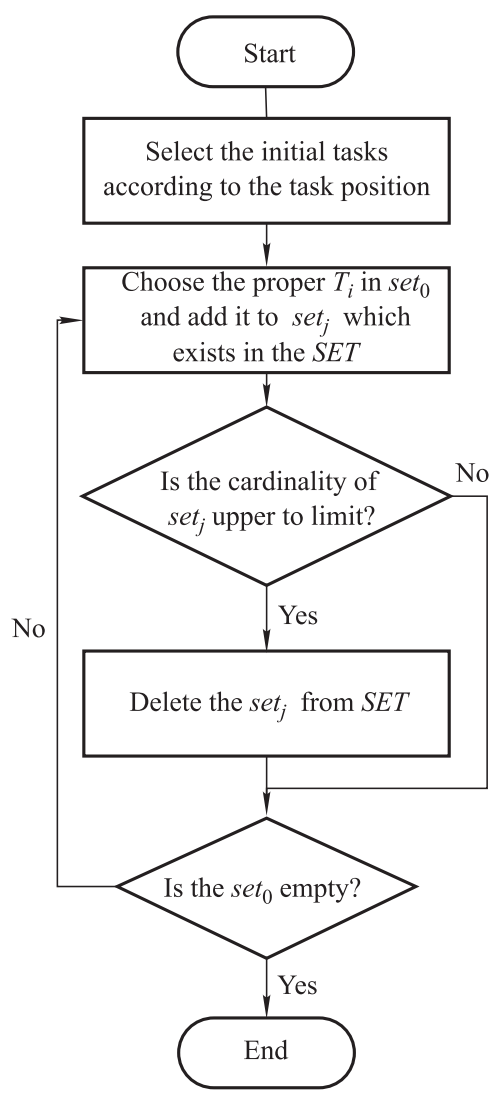

Fig. 2 Phases of the GCABTD algorithm

The implementations of the GCABTD can be described as follows:

Step 1 Select initial tasks. If $N_{T c}=2$, select two tasks as the initial task set initial, which has a maximum distance between each other; if $N_{T c}>2$, select two tasks as the initial task set initial which has a maximum distance between each other, then find the remain task which has a maximum sum of the distance to the tasks in the initial and put it in the initial until $\mid$ initial $\mid=N_{T c}$.

Step 2 Establish the task cluster set. Establish the task set $s_{0} t_{0}$, which including all the tasks. For every task $T I_{i}$ in initial, establish a task cluster set set $_{i}=\varnothing(i=$ $\left.1,2, \ldots, N_{T c}\right)$, and set $_{i}=$ set $_{i} \cup\left\{T I_{i}\right\}$, set se $_{0}=\frac{\text { set }_{0}}{\text { initial }_{\text {int }}}$. Establish the feasible task cluster set $S E T$ where $S E T=$ $\left\{\right.$ set $\left._{i} \mid i=1,2, \ldots, N_{T c}\right\}$.

Step 3 Task clustering. For the task $T_{i}\left(T_{i} \in\right.$ set $\left._{0}\right)$, calculate $d i s_{i, j}$, the sum of distance to the tasks in $\operatorname{set}_{j}\left(\operatorname{set}_{j} \in S E T\right)$. Solve the optimization problem 
$\min _{i, j} \operatorname{dis}_{*, *}$, and set $_{j}=\operatorname{set}_{j} \cup\left\{T_{i}\right\}$, set $_{0}=\frac{\text { set }_{0}}{\left\{T_{i}\right\}}$.

Step 4 The exit of the task cluster set. Calculate the cardinality of $\operatorname{set}_{j}\left(\right.$ set $\left._{j} \in S E T\right)$. If $\mid$ set $_{j} \mid=\left\lceil\frac{N_{T}}{N_{T c}}\right\rceil$, $S E T=S E T \backslash\left\{\operatorname{set}_{j}\right\}$ and go to Step 5; otherwise, go to Step 5 directly.

Step 5 Judge the termination condition of the algorithm. Calculate the cardinality of $\operatorname{set}_{0}$. If $\left|\operatorname{set}_{0}\right|>0$, go to Step 3; otherwise, end the algorithm.

\subsection{UAV allocation algorithm}

Problem 2 is a multi-objective integer programming problem, which is difficult to be solved by some existing heuristic algorithms. Considering that the NSGA-II algorithm [16-18] proposed in recent years can effectively solve such problems, we use the architecture of the NSGA-II algorithm for the reference and propose a multi-objective genetic algorithm based on the constraint violation degree (MOGABCVD). The proposed algorithm can effectively solve Problem 2, and the implementations are as follows:

(i) Code and decode. Chromosomes are encoded in the binary form, and $\boldsymbol{Y}_{i} \in \mathbf{R}^{N_{\mathrm{UAV}} N_{T c} \times 1}$ denotes a chromosome. Due to the decision variable, $\boldsymbol{y}$ is a matrix of $N_{\mathrm{UAV}} \times N_{T c}$, and the relationship between $\boldsymbol{y}$ and $\boldsymbol{Y}_{i}$ can be described as follows:

$$
\begin{gathered}
{\left[\boldsymbol{Y}_{i}\right]_{(j-1) N_{\mathrm{UAV}}+1: j N_{\mathrm{UAV}}, 1}=[\boldsymbol{y}]_{1: N_{\mathrm{UAV}}, J},} \\
j=1,2, \ldots, N_{T c} .
\end{gathered}
$$

(ii) Use genetic operators for population evolution. Genetic operators include the selection operator, crossover operator and mutation operator. In this algorithm, the selection operator adopts the binary tournament selection strategy, the crossover operator adopts the two-point crossover strategy, and the mutation operator adopts the uniform mutation strategy.

(iii) The non-dominated sorting of individuals within the population. Non-dominated sorting is an important way to judge individual fitness in multi-objective evolutionary algorithms. Assume that, at the instant $k$, the number of individuals in a population is $N_{0}$, and take the $N_{0}$ individuals as the parents, $N_{0}$ new individuals can be obtained by the genetic operators. At this moment, the number of individuals in a population is $2 N_{0}$. According to the principle of survival of the fittest, we need to sort out all the individuals in a non-dominated manner, so as to select the better individual to compose the next population.

Firstly, introduce the concept of the dominated. For the two individuals $\boldsymbol{Y}_{i}$ and $\boldsymbol{Y}_{j}$, if they satisfy that all the objectives of $\boldsymbol{Y}_{i}$ are not worse than those of $\boldsymbol{Y}_{j}$, and at least one objective of $\boldsymbol{Y}_{i}$ is better than that of $\boldsymbol{Y}_{j}, \boldsymbol{Y}_{j}$ is dominated by $\boldsymbol{Y}_{i}$, which is expressed as $\boldsymbol{Y}_{i} \prec \boldsymbol{Y}_{j}$. If $\boldsymbol{Y}_{j}$ is not dominated by $\boldsymbol{Y}_{i}$ and $\boldsymbol{Y}_{i}$ is not dominated by $\boldsymbol{Y}_{j}, \boldsymbol{Y}_{i}$, and $\boldsymbol{Y}_{j}$ are undifferentiated, which is expressed as $\boldsymbol{Y}_{i} \sim \boldsymbol{Y}_{j}$.

The non-dominated sorting uses the concept of dominance to divide all individuals into sets of different ranks. This division should satisfy that any two individuals in the same set are undifferentiated, and any individual with a low level can dominate all the individuals in a higher level set.

(iv) Rank the crowing distance [16]. After the nondominated sorting step, there are generally two strategies for selecting individuals to a new generation as shown in Fig. 3. First, individuals in a low-level group are all selected. When the last group that is allowed to be accommodated is considered, there exist more individuals than the space reserved in the new generation.

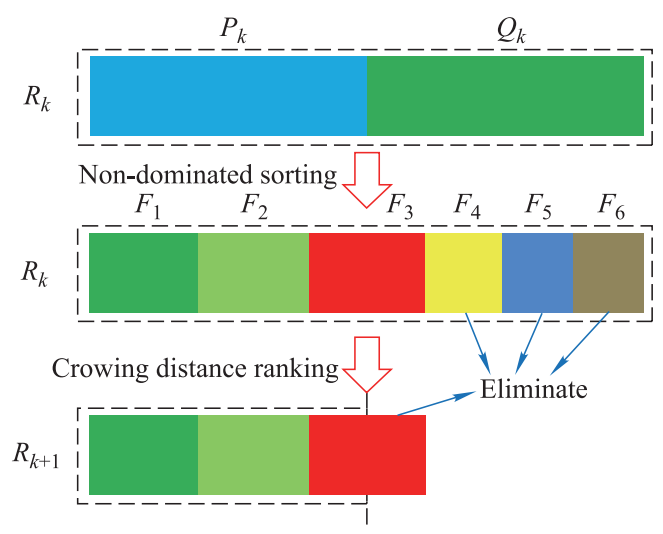

(a) Select the individuals only in a low level set

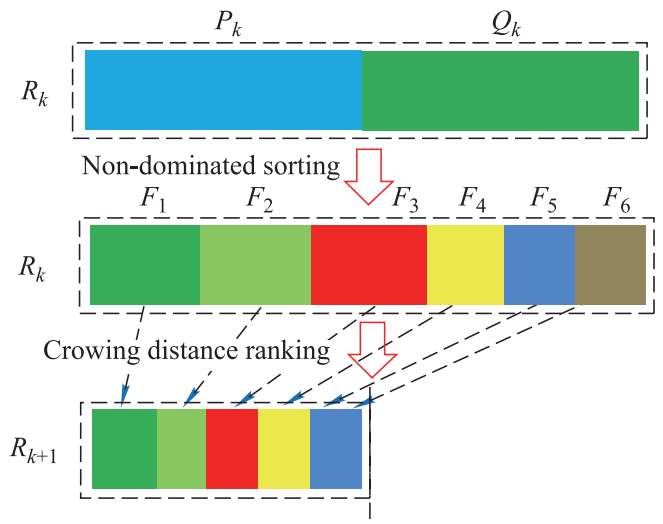

(b) Select the individuals in every set

Fig. 3 Two strategies for selecting individuals to the new generation

In order to maintain the diversity of the population, we need to sort out the crowding distances among the individuals and select the individuals with a larger crowding distance to enter the new generation, as shown in 
Fig. 3(a). The second is to rank the crowding distances of each level set first and then to tailor the individuals in each set according to the crowding distance so that the total number of individuals selected to the new generation is exactly $N_{0}$, as shown in Fig. 3(b).

For the individual $i$ in the set, the crowing distance can be defined as

$$
c d_{i}=\left\{\begin{array}{l}
\text { inf, } \exists j \in\left[1, N_{o b j}\right] ; \text { index }_{j}(i)=1 \text { or } N_{\text {set }} \\
\sum_{j=1}^{N_{\text {obj }}}\left(o b j_{\text {index }_{j}(i)+1}^{j}-o b j_{\text {index }_{j}(i)-1}^{j}\right), \quad \text { else }
\end{array}\right.
$$

where $N_{o b j}$ is the number of the optimization targets, $N_{\text {set }}$ is the cardinality of set, $\operatorname{index}_{j}(i)$ is the array number of the individual $i$ in the ascending order of the $j$ th target value and $o b j_{k}^{j}$ denotes the target value of the ranked number $k$ in the ascending order of the $j$ th target value. The crowding distance describes the individual contribution to the diversity of the population. Thus, the individual with the larger crowding distance should be retained in the new generation. Since the second method is better to maintain the diversity of the population than the first method, it is adopted in our algorithm.

In Problem 2, the obtained solution must satisfy the constraints (9), (10), and (11), thus we use the penalty function to add the constraint information to the objective function. For Problem 2, the constraint violation degree of the individual can be defined as follows:

$$
\begin{gathered}
G\left(\boldsymbol{Y}_{i}\right)=\frac{\delta_{1}}{N_{L} N_{T c}} \sum_{i=1}^{N_{L}} \sum_{j=1}^{N_{T c}} \max \left(-\frac{[\boldsymbol{R} \boldsymbol{d} \boldsymbol{t}]_{i, j}}{[\boldsymbol{R} \boldsymbol{l}]_{i, j}}, 0\right)+ \\
\frac{\delta_{2}}{N_{U} N_{T c}} \sum_{i=1}^{N_{U}} \sum_{j=1}^{N_{T c}} \max \left(\frac{\delta_{U}-[\boldsymbol{S} \boldsymbol{d}]_{i, j}}{\delta_{U}}, 0\right)+ \\
\frac{\delta_{3}}{N_{\mathrm{UAV}}} \sum_{i=1}^{N_{\mathrm{UAV}}} \max \left(\frac{\sum_{j=1}^{N_{T c}}[\boldsymbol{y}]_{i, j}-1}{\left.\sum_{j=1}^{N_{T c}}[\boldsymbol{y}]_{i, j}, 0\right)}\right. \\
\delta_{1}+\delta_{2}+\delta_{3}=1
\end{gathered}
$$

where $\delta_{1}, \delta_{2}$ and $\delta_{3}$ represent the corresponding weight of each constraint, they can be adjusted according to specific circumstances. It can be seen from (19) that $G\left(\boldsymbol{Y}_{i}\right) \in[0,1]$ and the greater the constraint violation degree of the individual is, the greater the penalty function value is. Also, the penalty function value is zero if the individual does not violate the constraint.

According to the definition of the constraint violation degree, the constraint information can be reflected in the optimization objective and Problem 2 can be transformed into the following problem.

\section{Problem 4}

$$
\begin{gathered}
\left\{\begin{array}{l}
\min \sum_{i=1}^{N_{L}} \sum_{j=1}^{N_{T c}}\left[\boldsymbol{A} \boldsymbol{l}^{\mathrm{T}} \boldsymbol{y}\right]_{i, j}+\alpha G(\cdot) \\
\min -A C C+\alpha G(\cdot)
\end{array}\right. \\
\text { s.t. }[y]_{i, j}=0 \text { or } 1, \quad i=1,2, \ldots, N_{\mathrm{UAV}} ; j=1,2, \ldots, N_{T c}
\end{gathered}
$$

where $\alpha$ is the penalty factor, usually choosing a larger value. The constant term $\boldsymbol{R} \boldsymbol{l}$ in the $R D T$ is omitted to simplify the description of problem. The $0-1$ constraint of decision variables can be guaranteed by selecting the suitable crossover and mutation operators. In each evolution, all individuals will be punished according to the extent of their constraint violations, and the individuals which violate the constraints can be eliminated gradually. Eventually, solutions with zero degree of the constraint violation will be achieved. The flowchart of the MOGABCVD algorithm is shown in Fig. 4.

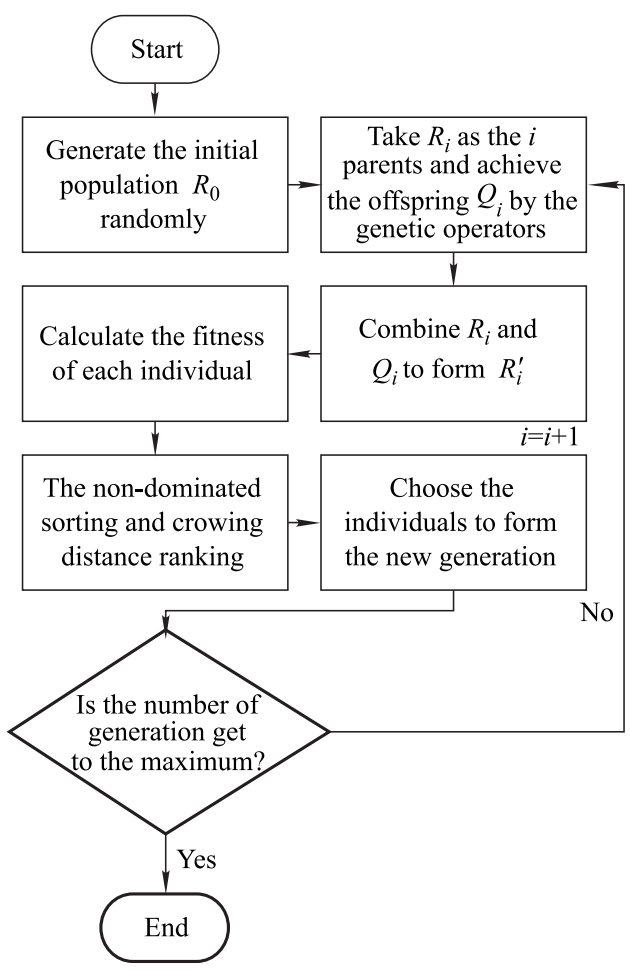

Fig. 4 Phases of the MOGABCVD algorithm

\subsection{MAV allocation algorithm}

As we can see that Problem 3 is an integer programming problem. Since it is an NP-hard problem, we cannot find the optimal solution to the problem in polynomial time. The common method to solve the integer programming problem is the branch-and-bound method, but the time 
complexity of the algorithm may increase exponentially with the increase of the problem scale. Although the evolutionary algorithm can solve this kind of problem in a relatively short period of time, the time cost is large. Therefore, we decompose the optimization problem into multiple suboptimization problems which are easy to be solved, and propose an MAV allocation algorithm with decomposition (MAVAAWD).

The main ideal of the decomposition is to assign the MAV into the UAV group in a particular sequence. Thus, we just need to allocate the available MAVs for one UAV group at one time. For the UAV group $j$, the optimization problem can be described as follows:

\section{Problem 5}

$$
\min _{z_{1}: N_{\mathrm{UAV}}, j} C c r_{j}=\sum_{i=1}^{N_{C}}\left[\boldsymbol{C a}^{\mathrm{T}} \boldsymbol{z}_{1: N_{\mathrm{UAV}}, j}\right]_{i, j}
$$

s.t.

$$
\begin{gathered}
{\left[\boldsymbol{C} \boldsymbol{a}^{\mathrm{T}} \boldsymbol{z}_{1: N_{\mathrm{UAV}}, j}-\boldsymbol{C r}_{1: N_{\mathrm{UAV}}, j}\right]_{i, j} \geqslant 0, \quad i=1,2, \ldots, N_{U}} \\
\sum_{k=1}^{j}[\boldsymbol{z}]_{i, k} \leqslant 1, \quad i=1,2, \ldots, N_{\mathrm{UAV}} \\
{[\boldsymbol{z}]_{i, j}=0 \text { or } 1, \quad i=1,2, \ldots, N_{\mathrm{UAV}} . \quad \text { (22) }}
\end{gathered}
$$

It should be noted that in order to facilitate the formulation of the optimization problem, the constant term $\boldsymbol{C} \boldsymbol{r}_{1: N_{\mathrm{UAV}}, j}$ in the objective function is omitted, and the optimization solution is the same as the original optimization problem. In (22), the second constraint ensures that one MAV is assigned to only one UAV group at most, and guarantees that the global solution satisfies the original constraints. At the same time, due to the requirement of the remained MAVs information by the second constraint, the sub-optimization problems must be solved in turn. The order of the assignment has an impact on the optimization solution (under certain distribution sequences, there may not exist the solution), thus we need to calculate the set perms and the total permutation of the UAV group sequence, before the decomposition of the optimization problem. Then randomly select an optimization sequence $s_{i}$ to solve the optimization problem. For all sub-optimization problems, if the solutions exist then the algorithm ends; if the solutions does not exist, $s_{i}$ will be deleted to form perms, and another optimization sequence will be selected in perms for optimization until all the sub-optimization problems can be solved.

If the optimal solutions are found for all the suboptimization problems under the sequence, the optimal solution of each sub-optimization problem can be combined to get the global solution of the optimization problem.

$$
\begin{gathered}
\widetilde{\boldsymbol{z}}=\left[\boldsymbol{z}_{1: N_{\mathrm{UAV}}, S_{1}}, \boldsymbol{z}_{1: N_{\mathrm{UAV}}, S_{2}}, \ldots, \boldsymbol{z}_{\left.1: N_{\mathrm{UAV}}, S_{N_{T c}}\right]}\right] \\
S_{i}=\underset{s}{\left.\arg \operatorname{serial}_{k}(s)=i\right),} \\
i=1,2, \ldots, N_{T c}
\end{gathered}
$$

The number of decision variables in the original problem is $N_{\mathrm{UAV}} N_{T c}$, and the number of decision variables in the sub-optimization problem after decomposition is $N_{\mathrm{UAV}}$. Although the number of optimization problems changes from 1 to $N_{T c}$, the time complexity increases linearly. Therefore, when the number of clusters is large, the scale of the problem can be significantly reduced. The flowchart of the MAVAAWD algorithm is shown in Fig. 5.

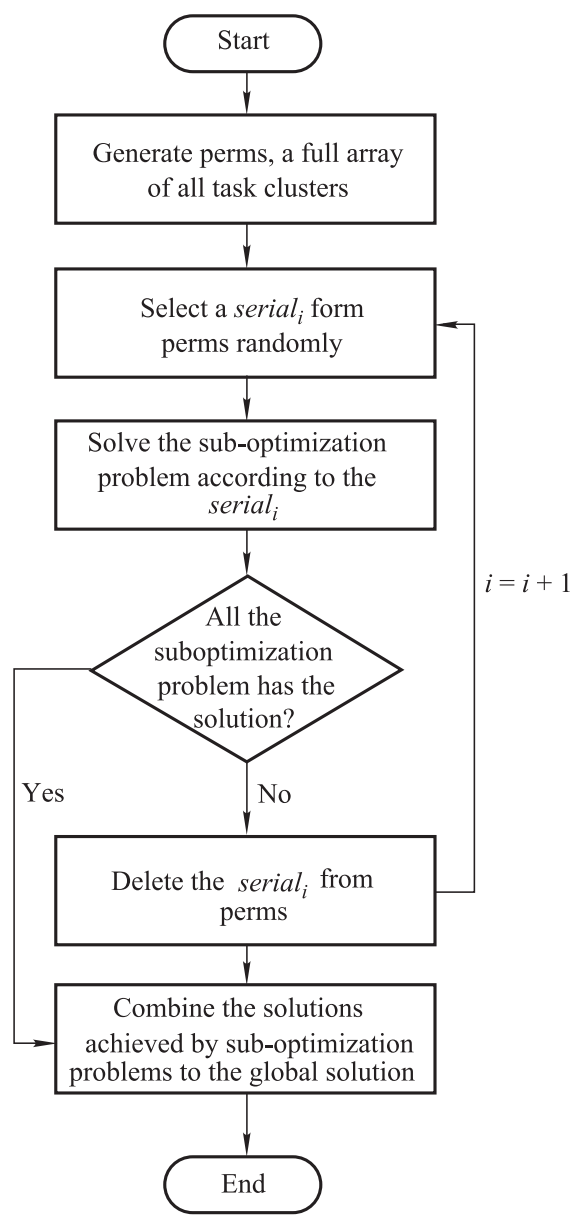

Fig. 5 Phases of the MAVAAWD algorithm

\section{Simulation}

The three algorithms discussed above will be simulated. We run the algorithms on a personal computer with pentium(R) dual-core CPU E6600 $3.06 \mathrm{GHz}$ and it is based on the Matlab R2009a software environment. 


\subsection{Phase 1}

There are 32 tasks and the position of each task can be seen in the Table 1. According to the operational plans, these tasks need to be divided into five clusters based on their positions.

Table 1 Position of each task

\begin{tabular}{ccc||ccc}
\hline Task & $X$ & $Y$ & Task & $X$ & $Y$ \\
\cline { 3 - 5 } 1 & 52.9263 & 37.8501 & 17 & 53.8303 & 43.1260 \\
2 & 59.2529 & 63.9443 & 18 & 19.5800 & 32.2012 \\
3 & 35.6086 & 62.4794 & 19 & 63.6900 & 23.8364 \\
4 & 95.6741 & 57.3491 & 20 & 98.7569 & 98.9637 \\
5 & 43.1887 & 10.4419 & 21 & 97.2006 & 26.7333 \\
6 & 89.8858 & 37.3027 & 22 & 69.4106 & 61.5073 \\
7 & 76.7491 & 20.3776 & 23 & 68.5559 & 99.8219 \\
8 & 74.0074 & 18.7913 & 24 & 11.8831 & 30.5921 \\
9 & 59.3561 & 27.9589 & 25 & 65.5319 & 70.4493 \\
10 & 92.5727 & 62.7326 & 26 & 92.9411 & 19.2665 \\
11 & 85.8205 & 42.4624 & 27 & 53.0874 & 9.5976 \\
12 & 54.8766 & 6.1576 & 28 & 51.0071 & 76.0051 \\
13 & 84.2079 & 44.4167 & 29 & 68.0715 & 37.5511 \\
14 & 30.7281 & 90.7258 & 30 & 86.9777 & 99.2231 \\
15 & 96.3042 & 26.5371 & 31 & 15.1486 & 46.0015 \\
16 & 24.8760 & 31.6084 & 32 & 32.6902 & 59.9421 \\
\hline
\end{tabular}

Using the parameters and the proposed GCABTD algorithm, we can achieve the clustering result as shown in Fig. 6.

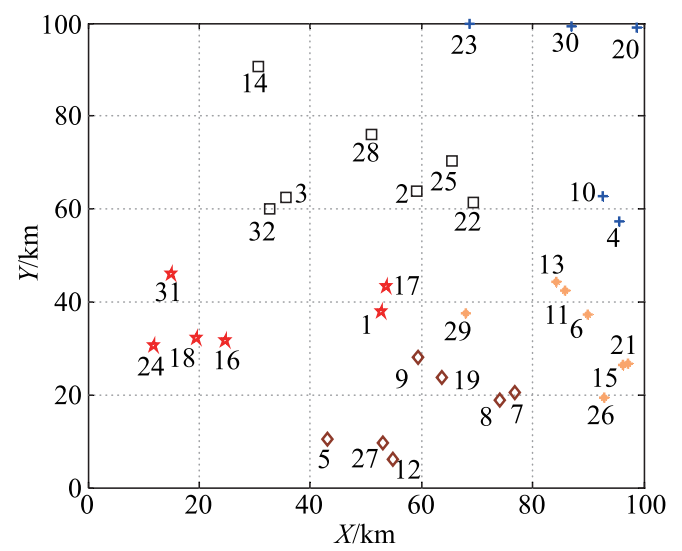

Fig. 6 Clustering result calculated by GCABTD algorithm

We can see directly in Fig. 6 that all tasks are divided into five clusters according to their position. The result can be described in Table 2.

Table 2 Clustering result calculated by GCABTD algorithm

\begin{tabular}{cc}
\hline Cluster & Tasks \\
\hline 1 & $\mathrm{~T}_{4}, \mathrm{~T}_{10}, \mathrm{~T}_{20}, \mathrm{~T}_{23}, \mathrm{~T}_{30}$ \\
2 & $\mathrm{~T}_{6}, \mathrm{~T}_{11}, \mathrm{~T}_{13}, \mathrm{~T}_{15}, \mathrm{~T}_{21}, \mathrm{~T}_{26}, \mathrm{~T}_{29}$ \\
3 & $\mathrm{~T}_{5}, \mathrm{~T}_{7}, \mathrm{~T}_{8}, \mathrm{~T}_{9}, \mathrm{~T}_{12} \mathrm{~T}_{19}, \mathrm{~T}_{27}$ \\
4 & $\mathrm{~T}_{1}, \mathrm{~T}_{16}, \mathrm{~T}_{17}, \mathrm{~T}_{18}, \mathrm{~T}_{24}, \mathrm{~T}_{31}$ \\
5 & $\mathrm{~T}_{2}, \mathrm{~T}_{3}, \mathrm{~T}_{14}, \mathrm{~T}_{22}, \mathrm{~T}_{25}, \mathrm{~T}_{28}, \mathrm{~T}_{32}$ \\
\hline
\end{tabular}

To illustrate the superiority of the GCABTD algorithm, we compare it with the GCWOICC algorithm [8] and the $k$-means algorithm [15]. In the simulation, the number of tasks and the number of task clusters remain unchanged, the geographic positions of the tasks are randomly generated in the same geographical area, and the task distance within the cluster is used as an evaluation index. After 1000 runs, the comparison of each algorithm is shown in Table 3.

Table 3 Clustering result of algorithms

\begin{tabular}{cccc}
\hline Method & Mean & Variance & Time/s \\
\hline GCBTD & 452.43 & 2147.23 & 0.0136 \\
$k$-means & 471.77 & 6838.63 & 0.0016 \\
GCWOICC & 460.74 & 2389.79 & 0.0106 \\
\hline
\end{tabular}

As can be seen from Table 3, the GCABTD is superior to the $k$-means and the GCWOICC algorithms in terms of the average of evaluation indices, and can obtain a clustering plan with smaller task distances within the cluster. The variance of the evaluation indices is smaller than that of the $k$-means algorithm and the GCWOICC algorithm, indicating that its stability is stronger. Although the GCABTD algorithm pays more for its time, the time overhead of the algorithm is within $0.1 \mathrm{~s}$, which is totally acceptable.

To exhibit the advantage of the proposed algorithms for the large-scale problem, we compare the three clustering algorithms with different numbers of tasks. After 100 runs, the result is shown in Fig. 7.

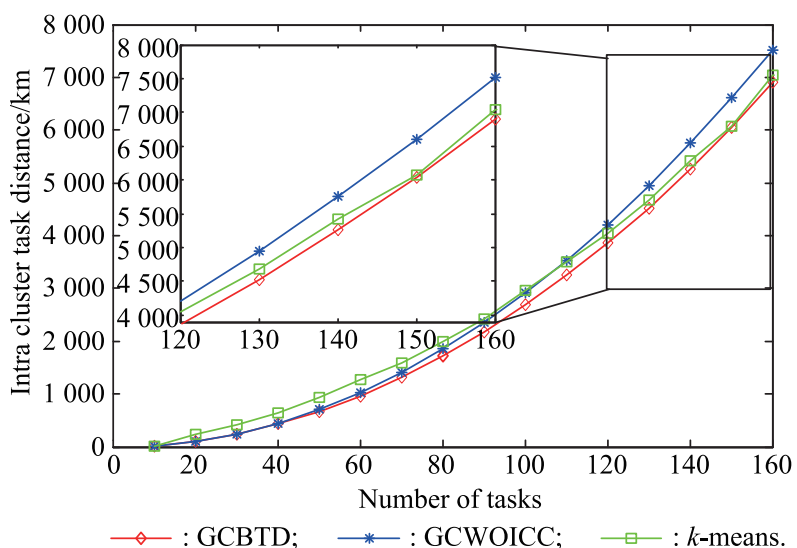

Fig. 7 Intra cluster task distance of each algorithm with different numbers of tasks

As we can see in Fig. 7, when the number of tasks is around 100 , the $k$-means algorithm and the GCWOICC algorithm get the similar preference. The $k$-means algorithm can achieve a better preference than the GCWOICC algorithm when the number is more than 100 . At the same time, the proposed GCABTD algorithm can always keep a lower intra cluster task distance, when the number of tasks is between 10 and 160 . 


\subsection{Phase 2}

To execute the tasks in Phase 1, 30 UAVs can be chosen. The constrained resources $\mathrm{CR}$ and unconstrained resources UR of each UAV are shown in Table 4 and Table 5.

Table 4 Constrained resources of each UAV

\begin{tabular}{cccccccccccc}
\hline UAV & $\mathrm{CR}_{1}$ & $\mathrm{CR}_{2}$ & $\mathrm{CR}_{3}$ & $\mathrm{CR}_{4}$ & $\mathrm{CR}_{5}$ & $\mathrm{UAV}$ & $\mathrm{CR}_{1}$ & $\mathrm{CR}_{2}$ & $\mathrm{CR}_{3}$ & $\mathrm{CR}_{4}$ & $\mathrm{CR}_{5}$ \\
\hline 1 & 1 & 6 & 9 & 7 & 2 & 16 & 6 & 9 & 6 & 9 & 3 \\
2 & 8 & 3 & 0 & 0 & 9 & 17 & 3 & 1 & 3 & 8 & 3 \\
3 & 3 & 5 & 7 & 8 & 4 & 18 & 2 & 5 & 7 & 6 & 2 \\
4 & 9 & 8 & 7 & 4 & 0 & 19 & 4 & 3 & 5 & 2 & 3 \\
5 & 1 & 9 & 7 & 3 & 2 & 20 & 8 & 9 & 7 & 0 & 1 \\
6 & 4 & 3 & 3 & 2 & 9 & 21 & 6 & 3 & 7 & 9 & 8 \\
7 & 8 & 2 & 1 & 5 & 0 & 22 & 8 & 7 & 4 & 7 & 3 \\
8 & 6 & 9 & 0 & 2 & 8 & 23 & 6 & 8 & 8 & 9 & 0 \\
9 & 0 & 7 & 8 & 5 & 6 & 24 & 4 & 6 & 2 & 1 & 7 \\
10 & 8 & 5 & 2 & 1 & 1 & 25 & 3 & 3 & 4 & 6 & 2 \\
11 & 4 & 7 & 5 & 9 & 9 & 26 & 4 & 6 & 3 & 7 & 9 \\
12 & 0 & 8 & 7 & 7 & 1 & 27 & 6 & 9 & 2 & 3 & 5 \\
13 & 7 & 2 & 4 & 5 & 2 & 28 & 8 & 4 & 6 & 8 & 7 \\
14 & 0 & 2 & 2 & 8 & 6 & 29 & 7 & 9 & 1 & 0 & 5 \\
15 & 7 & 7 & 5 & 6 & 2 & 30 & 2 & 6 & 7 & 1 & 4 \\
\hline
\end{tabular}

Table 5 Unconstrained resources of each UAV

\begin{tabular}{ccccccccccccc}
\hline $\mathrm{UAV}$ & $\mathrm{UR}_{1}$ & $\mathrm{UR}_{2}$ & $\mathrm{UR}_{3}$ & $\mathrm{UR}_{4}$ & $\mathrm{UR}_{5}$ & $\mathrm{UAV}$ & $\mathrm{UR}_{1}$ & $\mathrm{UR}_{2}$ & $\mathrm{UR}_{3}$ & $\mathrm{UR}_{4}$ & $\mathrm{UR}_{5}$ \\
\hline 1 & 0 & 5 & 9 & 2 & 5 & 16 & 7 & 1 & 3 & 7 & 9 \\
2 & 1 & 3 & 1 & 2 & 7 & 17 & 8 & 2 & 0 & 7 & 6 \\
3 & 6 & 2 & 8 & 0 & 1 & 18 & 3 & 0 & 1 & 2 & 4 \\
4 & 4 & 9 & 0 & 6 & 1 & 19 & 2 & 9 & 6 & 5 & 3 \\
5 & 1 & 4 & 5 & 4 & 0 & 20 & 5 & 0 & 2 & 0 & 4 \\
6 & 9 & 3 & 8 & 9 & 3 & 21 & 1 & 2 & 2 & 4 & 8 \\
7 & 6 & 1 & 1 & 4 & 2 & 22 & 5 & 3 & 3 & 0 & 7 \\
8 & 4 & 9 & 7 & 3 & 1 & 23 & 1 & 6 & 4 & 9 & 9 \\
9 & 8 & 1 & 8 & 2 & 9 & 24 & 2 & 8 & 0 & 1 & 2 \\
10 & 2 & 0 & 1 & 2 & 3 & 25 & 8 & 6 & 3 & 4 & 6 \\
11 & 4 & 6 & 3 & 8 & 8 & 26 & 5 & 3 & 9 & 9 & 5 \\
12 & 2 & 0 & 6 & 3 & 7 & 27 & 9 & 7 & 4 & 0 & 5 \\
13 & 3 & 8 & 1 & 2 & 7 & 28 & 9 & 6 & 1 & 0 & 1 \\
14 & 9 & 9 & 6 & 0 & 8 & 29 & 1 & 0 & 8 & 4 & 4 \\
15 & 5 & 7 & 0 & 9 & 5 & 30 & 5 & 2 & 8 & 9 & 3 \\
\hline
\end{tabular}

The constrained and unconstrained resources requirements of tasks in each task cluster are shown in Table 6.

Table 6 Constrained and unconstrained resources of each task cluster

\begin{tabular}{ccccccccccc}
\hline Cluster & $\mathrm{CR}_{1}$ & $\mathrm{CR}_{2}$ & $\mathrm{CR}_{3}$ & $\mathrm{CR}_{4}$ & $\mathrm{CR}_{5}$ & $\mathrm{UR}_{1}$ & $\mathrm{UR}_{2}$ & $\mathrm{UR}_{3}$ & $\mathrm{UR}_{4}$ & $\mathrm{UR}_{5}$ \\
\hline 1 & 12 & 12 & 13 & 12 & 8 & 12 & 7 & 9 & 6 & 12 \\
2 & 10 & 17 & 11 & 13 & 13 & 13 & 9 & 12 & 12 & 3 \\
3 & 21 & 14 & 9 & 11 & 11 & 13 & 11 & 18 & 15 & 15 \\
4 & 9 & 18 & 15 & 12 & 17 & 9 & 12 & 12 & 16 & 10 \\
5 & 18 & 18 & 18 & 13 & 18 & 14 & 11 & 18 & 21 & 13 \\
\hline
\end{tabular}

To demonstrate the advantage of the MOGABCVD algorithm, we compare it with the MOPSO algorithm [19] for this problem. Parameters setting in the MOGABCVD algorithm are listed as follows: $\delta_{1}=\delta_{2}=\delta_{3}=\frac{1}{3}$, $\alpha=10000, \delta_{U}=0.5$. For the two algorithms, the population size is 100 and the number of iterations is 300 . The Pareto front of two algorithms is shown in Fig. 8.

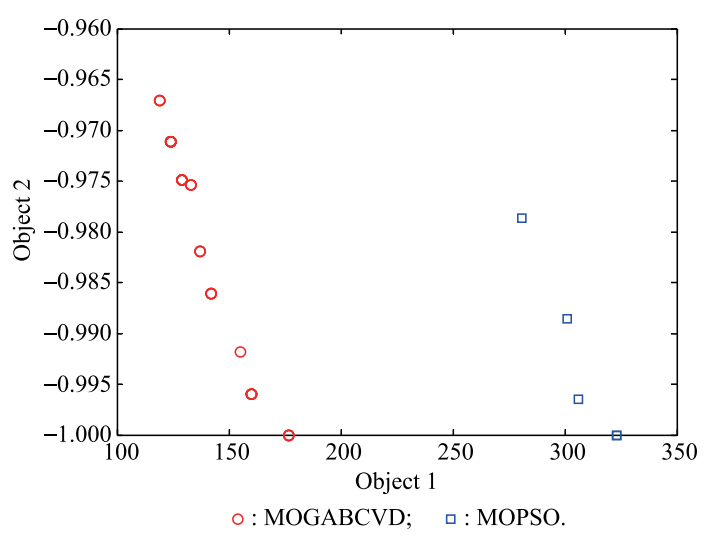

Fig. 8 Pareto front of two algorithms

It can be seen from Fig. 8 that the Pareto front obtained by the proposed MOGABCVD is obviously superior to the MOPSO algorithm. Because the lack of the mutation operator in the MOPSO algorithm, the solution obtained is easy to fall into the local optimum. However, the proposed algorithm is based on the NSGA-II architecture, individuals in the population will oscillate at the local optimum after being manipulated by the mutation operator, and a better solution can be achieved in this way.

In order to further reflect the superiority of the penalty function, we compare it with the classical penalty function. The C-index, the S-index and the MS-index [20] are used to measure the Pareto fronts. The resource requirements matrix of task clusters and the UAV resource matrix are randomly generated, and other parameters remain unchanged. The average indexes obtained after 500 runs are shown in Table 7.

Table 7 Results under two penalty function

\begin{tabular}{cccc}
\hline & C-index & S-index & MS-index \\
\hline PFWCVD & 0.5517 & 0.7222 & 149.5616 \\
\hline PFWRS & 0.3936 & 0.7235 & 144.6104 \\
\hline
\end{tabular}

It can be seen from Table 7, for the S-index, algorithms with two penalty functions have a similar preference. But for the C-index and the MS-index, our penalty function is obviously superior to the penalty function with the refusal strategy. The penalty function with the refusal strategy gives an enormous penalty to the individual who violates the constraint. Although this is theoretically feasible, it is easy to reduce the diversity of solutions in the actual process, and a low quality solution set will be achieved. The proposed penalty function is based on the degree of the individual constraint violation. It can effectively avoid the arbitrary abandonment of individuals who violate the 
constraint but have great potential, and can increase the diversity of solution sets probably.

Table 8 shows the performance of two penalty functions under different scales. The proposed penalty function has a better performance than the PFWRS in the C-index and MS-index. Although, for the S-index, which is used to describe the homogeneity of all non-dominated solutions, the PFWCVD performs a little worse than the PFWRS, it will not affect the quality of the single solution which may be selected for the final scheme.

Table 8 Performance of two penalty functions under different scales

\begin{tabular}{ccccc}
\hline$\left(N_{T}, N_{\mathrm{UAV}}\right)$ & Algorithm & C-index & S-index & MS-index \\
\hline \multirow{2}{*}{$(50,50)$} & PFWCVD & 0.4650 & 0.7714 & 38.7919 \\
& PFWRS & 0.2216 & 0.5590 & 22.3800 \\
\hline \multirow{2}{*}{$(70,70)$} & PFWCVD & 0.6314 & 0.7227 & 47.8853 \\
& PFWRS & 0.1307 & 0.5103 & 23.2002 \\
\hline \multirow{2}{*}{$(90,90)$} & PFWCVD & 0.6044 & 0.7499 & 71.1137 \\
& PFWRS & 0.1134 & 0.5689 & 25.7811 \\
\hline
\end{tabular}

\subsection{Phase 3}

To satisfy the command and control ability requirement of the UAV, the MAV should be allocated to the UAV group. The requirement BR of each UAV and the command and control ability CB of the MAV can be found in Table 9 and Table 10 .

Table 9 Command and control ability requirement of UAV

\begin{tabular}{ccccccccccccc}
\hline $\mathrm{UAV}$ & $\mathrm{BR}_{1}$ & $\mathrm{BR}_{2}$ & $\mathrm{BR}_{3}$ & $\mathrm{BR}_{4}$ & $\mathrm{BR}_{5}$ & $\mathrm{UAV}$ & $\mathrm{BR}_{1}$ & $\mathrm{BR}_{2}$ & $\mathrm{BR}_{3}$ & $\mathrm{BR}_{4}$ & $\mathrm{BR}_{5}$ \\
\hline 1 & 3 & 0 & 0 & 3 & 0 & 16 & 2 & 0 & 2 & 0 & 0 \\
2 & 2 & 2 & 1 & 2 & 2 & 17 & 3 & 0 & 1 & 0 & 0 \\
3 & 2 & 3 & 1 & 0 & 3 & 18 & 1 & 0 & 0 & 0 & 0 \\
4 & 2 & 3 & 1 & 2 & 0 & 19 & 2 & 2 & 1 & 2 & 0 \\
5 & 0 & 1 & 2 & 0 & 3 & 20 & 2 & 0 & 2 & 1 & 0 \\
6 & 1 & 2 & 0 & 0 & 3 & 21 & 1 & 1 & 0 & 2 & 0 \\
7 & 3 & 3 & 3 & 1 & 0 & 22 & 0 & 2 & 0 & 2 & 0 \\
8 & 1 & 3 & 0 & 0 & 0 & 23 & 1 & 1 & 0 & 3 & 0 \\
9 & 0 & 1 & 2 & 3 & 2 & 24 & 3 & 0 & 2 & 0 & 1 \\
10 & 3 & 0 & 0 & 3 & 3 & 25 & 0 & 1 & 3 & 2 & 1 \\
11 & 1 & 2 & 0 & 0 & 1 & 26 & 2 & 2 & 0 & 0 & 0 \\
12 & 2 & 0 & 2 & 0 & 0 & 27 & 3 & 3 & 1 & 0 & 0 \\
13 & 1 & 1 & 3 & 3 & 0 & 28 & 1 & 0 & 3 & 3 & 0 \\
14 & 3 & 3 & 0 & 0 & 2 & 29 & 2 & 1 & 0 & 0 & 1 \\
15 & 1 & 2 & 1 & 2 & 1 & 30 & 1 & 1 & 1 & 0 & 1 \\
\hline
\end{tabular}

Table 10 Command and control ability of MAV

\begin{tabular}{cccccccccccc}
\hline MAV & $\mathrm{CB}_{1}$ & $\mathrm{CB}_{2}$ & $\mathrm{CB}_{3}$ & $\mathrm{CB}_{4}$ & $\mathrm{CB}_{5}$ & $\mathrm{MAV}$ & $\mathrm{CB}_{1}$ & $\mathrm{CB}_{2}$ & $\mathrm{CB}_{3}$ & $\mathrm{CB}_{4}$ & $\mathrm{CB}_{5}$ \\
\hline 1 & 5 & 2 & 6 & 2 & 8 & 6 & 7 & 4 & 6 & 0 & 5 \\
2 & 8 & 4 & 1 & 6 & 3 & 7 & 2 & 2 & 0 & 3 & 1 \\
3 & 7 & 9 & 6 & 8 & 4 & 8 & 0 & 3 & 5 & 5 & 8 \\
4 & 4 & 5 & 6 & 7 & 6 & 9 & 5 & 7 & 5 & 0 & 5 \\
5 & 3 & 9 & 6 & 6 & 9 & 10 & 8 & 9 & 8 & 8 & 5 \\
\hline
\end{tabular}

Choose a typical solution (the objectives of problem 4 is 137 and -0.981 9) in Phase 2 which is shown in Table 11.
Table 11 Typical solution in situation 2

\begin{tabular}{cc}
\hline Cluster & $\mathrm{UAV}$ \\
\hline 1 & $\mathrm{U}_{20}, \mathrm{U}_{21}, \mathrm{U}_{25}$ \\
2 & $\mathrm{U}_{3}, \mathrm{U}_{10}, \mathrm{U}_{11}, \mathrm{U}_{17}$ \\
3 & $\mathrm{U}_{1}, \mathrm{U}_{8}, \mathrm{U}_{19}, \mathrm{U}_{26}, \mathrm{U}_{27}$ \\
4 & $\mathrm{U}_{5}, \mathrm{U}_{6}, \mathrm{U}_{18}, \mathrm{U}_{24}$ \\
5 & $\mathrm{U}_{2}, \mathrm{U}_{7}, \mathrm{U}_{9}, \mathrm{U}_{13}, \mathrm{U}_{30}$ \\
\hline
\end{tabular}

According to Table 9, Table 10 and Table 11, an MAV allocation plan (the objective of Problem 5 is 213) can be achieved by using the MAVAAWD, as shown in Table 12.

Table 12 MAV allocation plan achieve by MAVAAWD algorithm

\begin{tabular}{cc}
\hline UAVs & MAV \\
\hline 1 & $\mathrm{M}_{5}$ \\
2 & $\mathrm{M}_{4}, \mathrm{M}_{6}$ \\
3 & $\mathrm{M}_{2}, \mathrm{M}_{9}$ \\
4 & $\mathrm{M}_{1}, \mathrm{M}_{7}$ \\
5 & $\mathrm{M}_{3}, \mathrm{M}_{8}$ \\
\hline
\end{tabular}

To exhibit the effectiveness and practicability of the MAVAAWD, we compare it with the global branch and bound algorithm and the GA. The result of the MonteCarlo simulation (with 100 runs) is shown in Fig. 9.

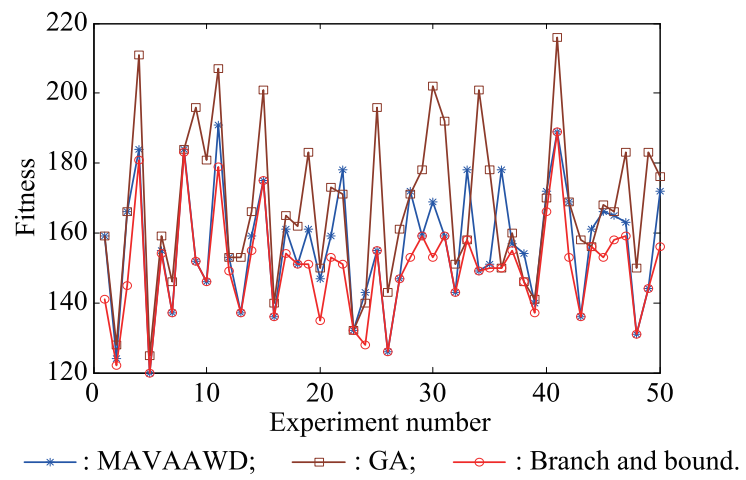

Fig. 9 RMSE for different MAV allocation algorithms

It can be seen from Fig. 9, the quality of the solutions obtained by the MAVAAWD is significantly better than that of the GA. At the same time, these two algorithms cannot guarantee the global optimality of their solutions, thus they perform worse than the global branch and bound method in the mean fitness index. However, we can see in Table 13, the time cost of the global branch and bound method is larger and greater than that of the MAVAAWD and the GA, and the variance of the running time is also very large. Therefore, it is difficult to estimate the running time required by the practical application. The running time of the MAVAAWD is shorter than that of the GA algorithm, and the obtained fitness value is also superior to that of the GA algorithm. It should be noted here that if the GA algorithm continues to run for more generations, it may be possible 
to get a better solution than the MAVAAWD, but the time efficiency will be unacceptable.

Table 13 Results for different MAV allocation algorithms

\begin{tabular}{cccc}
\hline Method & $\begin{array}{c}\text { Fitness } \\
(\text { mean })\end{array}$ & $\begin{array}{c}\text { Time/s } \\
(\text { mean })\end{array}$ & $\begin{array}{c}\text { Time/s } \\
\text { (variance) }\end{array}$ \\
\hline MAVAAWD & 156.3000 & 0.1119 & $3.5991 \mathrm{e}-04$ \\
GA & 167.4800 & 5.3361 & 1.0402 \\
Branch and bound & 150.1600 & 75.4733 & $4.5630 \mathrm{e}+03$ \\
\hline
\end{tabular}

Finally, we compare the MAVAAWD and the GA with different numbers of MVAs (with 100 runs). The fitness and the time cost of each algorithm are shown in Fig. 10 and Table 14. It shows that the MAVAAWD can get a better solution in a short time. Also, when the number of MAVs continues to increase, the time cost of the MAVAAWD will also increase rapidly. Fortunately, the number of MAVs will not be too large in practice and the time cost of the MAVAAWD will be acceptable.

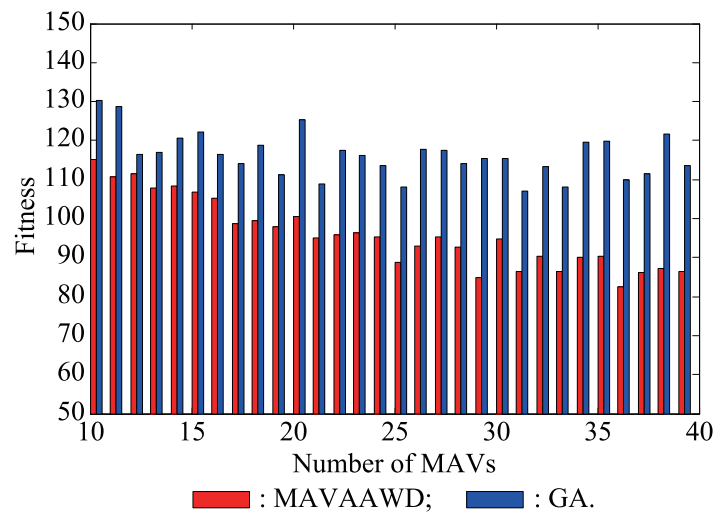

Fig. 10 Fitness of each algorithm with different number of MAVs

Table 14 Time cost of each algorithm with different numbers of MAVs

\begin{tabular}{ccccccc}
\hline Method & 15 & 20 & 25 & 30 & 35 & 40 \\
\hline MAVAAWD & 0.14 & 0.18 & 0.23 & 0.45 & 0.66 & 0.82 \\
\hline GA & 4.42 & 7.05 & 8.59 & 11.13 & 13.59 & 16.14 \\
\hline
\end{tabular}

Table 15 Final task coalition formation plan

\begin{tabular}{|c|c|c|c|}
\hline Cluster & Tasks & UAV & MAV \\
\hline 1 & $\begin{array}{c}\mathrm{T}_{4}, \mathrm{~T}_{10}, \mathrm{~T}_{20} \\
\mathrm{~T}_{23}, \mathrm{~T}_{30}\end{array}$ & $\mathrm{U}_{20}, \mathrm{U}_{21}, \mathrm{U}_{25}$ & $\mathrm{M}_{5}$ \\
\hline 2 & $\begin{array}{c}\mathrm{T}_{6}, \mathrm{~T}_{11}, \mathrm{~T}_{13}, \mathrm{~T}_{15}, \\
\mathrm{~T}_{21}, \mathrm{~T}_{26}, \mathrm{~T}_{29}\end{array}$ & $\mathrm{U}_{3}, \mathrm{U}_{10}, \mathrm{U}_{11}, \mathrm{U}_{17}$ & $\mathrm{M}_{4}, \mathrm{M}_{6}$ \\
\hline 3 & $\begin{array}{l}\mathrm{T}_{5}, \mathrm{~T}_{7}, \mathrm{~T}_{8}, \mathrm{~T}_{9} \\
\mathrm{~T}_{12} \mathrm{~T}_{19}, \mathrm{~T}_{27}\end{array}$ & $\begin{array}{c}\mathrm{U}_{1}, \mathrm{U}_{8}, \mathrm{U}_{19} \\
\mathrm{U}_{26}, \mathrm{U}_{27}\end{array}$ & $\mathrm{M}_{2}, \mathrm{M}_{9}$ \\
\hline 4 & $\begin{array}{l}\mathrm{T}_{1}, \mathrm{~T}_{16}, \mathrm{~T}_{17}, \\
\mathrm{~T}_{18}, \mathrm{~T}_{24}, \mathrm{~T}_{31}\end{array}$ & $\mathrm{U}_{5}, \mathrm{U}_{6}, \mathrm{U}_{18}, \mathrm{U}_{24}$ & $\mathrm{M}_{1}, \mathrm{M}_{7}$ \\
\hline 5 & $\begin{array}{c}\mathrm{T}_{2}, \mathrm{~T}_{3}, \mathrm{~T}_{14}, \mathrm{~T}_{22}, \\
\mathrm{~T}_{25}, \mathrm{~T}_{28}, \mathrm{~T}_{32}\end{array}$ & $\begin{array}{c}\mathrm{U}_{2}, \mathrm{U}_{7}, \mathrm{U}_{9}, \\
\mathrm{U}_{13}, \mathrm{U}_{30}\end{array}$ & $\mathrm{M}_{3}, \mathrm{M}_{8}$ \\
\hline
\end{tabular}

Table 15 shows the final plan of the task coalition formation calculated through three phases. It can be verified that under this formation plan, all the tasks in the task clusters can be well executed and the demand for command and decision capability of the UAV in the task coalition can be fully satisfied.

\section{Conclusions}

The task coalition which is formed by the MAV and the UAV can execute complex tasks on the battlefield. In order to effectively reduce the scale of the formation problem, this paper fully considers the task geographical location, task resource requirements and the demand of the UAV for command and control ability, then divides the MAV/UAV task coalition formation process into three phases. For every phase, the effective algorithm is proposed, and the simulation results reveal that the proposed method can give a reasonable task coalition formation plan according to the battlefield situation. Future work is to study the task assignment in the task cluster and the dynamic adjustment of the formation plan when the situation is changed.

\section{References}

[1] MANATHARA J G, SUJIT P B, BEARD R W. Multiple UAV coalitions for a search and prosecute mission. Journal of Intelligent and Robotic Systems, 2011, 62(1): 125-158.

[2] ARSLAN O, ARMAGAN B, INALHAN G. Development of a mission simulator for design and testing of $\mathrm{C} 2$ algorithms and HMI concepts across real and virtual manned-unmanned fleets. Lecture Notes in Control and Information Sciences, 2009, 381(1): $431-458$.

[3] GARCIA R D, BARNES L, FIELDS M. Unmanned aircraft system as wingmen. Journal of Defense Modeling and Simulation, 2012, 9(1): 5- 15.

[4] HU X, YANG L Y, ZHANG J. The design and analysis of hierarchical decision-making for manned/unmanned cooperative engagement. Proc. of the 34th Chinese Control Conference, 2015: 2698-2703.

[5] CHEN C, ZHANG X W, XU J, et al. Human/unmanned-aerialvehicle team collaborative decision-making with limited intervention. Acta Aeronautica et Astronautica Sinica, 2015, 69(11): $3652-3665$.

[6] LIU Y F, ZHANG A. Cooperative task assignment method of manned/unmanned aerial vehicle formation. Systems Engineering and Electronics, 2010, 32(3): 584 - 588. (in Chinese)

[7] CHOI H, BRUNET L, HOW J P. Consensus-based decentralized auctions for robust task allocation. IEEE Trans. on Robotics, 2009, 25(4): 912 - 926.

[8] ZHONG Y, YAO P Y, SUN Y, et al. Cooperative task allocation method of MCAV/ UCAV formation. Mathematical Problems in Engineering, 2016, 1(1): 1-9.

[9] ZHANG Y, PARKER L E. Considering inter-task resource constraints in task allocation. Autonomous Agents and MultiAgent Systems, 2013, 26(3): 389-419.

[10] HU X X, MA H W, YE Q S, et al. Hierarchical method of task assignment for multiple cooperating UAV teams. Journal of Systems Engineering and Electronics, 2015, 26(5): 10001009.

[11] ZHANG Y Z, XIE S Y, ZHANG L, et al. Optimal task decision-making for heterogeneous multi-UAV cooperation reconnaissance. Journal of Northwestern Polytechnical University, 2017, 35(3): 386-392. (in Chinese) 
[12] SERVICE T C, ADAMS J A. Coalition formation for task allocation: theory and algorithms. Auton Agent and Multi-Agent Systerms, 2011, 22(2): 225-248.

[13] HAN B W, YAO P Y. Coalition formation of manned/unmanned aerial vehicle cluster based on Holon organization. Systems Engineering and Electronics, 2018, 40(1): 91-97. (in Chinese)

[14] ZHONG Y, YAO P Y, SUN Y, et al. Research on phasedforming method of manned/unmanned aerial vehicle task coalition. Systems Engineering and Electronics, 2017, 39(9): 2031-2038. (in Chinese)

[15] CUELL C, BONSAL B. An assessment of climatological synoptic typing by principal component analysis and $k$-means clustering. Theoretical and Applied Climatology, 2009, 98(34): $361-373$

[16] HONDA S, IGARASHI T, NARITA Y. Multi-objective optimization of curvilinear fiber shapes for laminated composite plates by using NSGA-II. Composites Part B: Engineering, 2013, 45(1): $1071-1078$.

[17] GHOLAMI M H, AZIZI M R. Constrained grinding optimization for time, cost, and surface roughness using NSGA-II. The International Journal of Advanced Manufacturing Technology, 2014, 73(5-8): $981-988$.

[18] ARUNACHALAM A, NAGARAJAN N P, MOHAN V, et al. Resolving team selection in agile development using NSGA-II algorithm. CSI Trans. on ICT, 2016, 4(2-4): 83-86.

[19] COELLO C, LECHUNGA S. MOPSO: a proposal for multiple objective particles warm optimization. Proc. of the IEEE Congress on Evolutionary Computation, 2002: 1050-1056.

[20] LI M Q, ZHENG J H. An indicator for assessing the spread of solutions in multi-objective evolutionary algorithm. Chinese Journal of Computers, 2011, 34(4): 647-664. (in Chinese)

\section{Biographies}

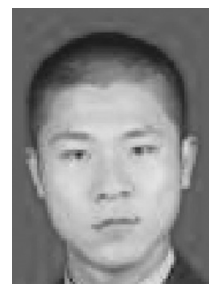

JIAO Zhiqiang was born in 1992. He received his B.S. degree in information and communication engineering from Air Force Engineering University in 2014, and his M.S. degree in information fusion from Air Force Engineering University in 2017. He is currently a Ph.D. candidate of Air Force Engineering University. His research interests include information fusion, command information system, and mission planning.

E-mail: jzq_paper@163.com

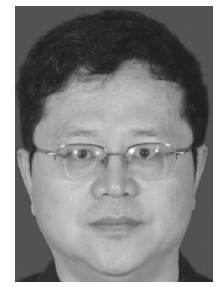

YAO Peiyang was born in 1960. He received his B.S. degree in 1982, and his M.S. degree in 1991 from Xidian University. Currently he is a professor in Information and Navigation College, Air Force Engineering University. His research interests include command and control theory and command automation system.

E-mail: ypy_664@163.com

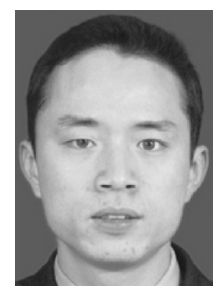

ZHANG Jieyong was born in 1983. He received his B.S. degree in 2006, his M.S. degree in 2008 and his Ph.D. degree in 2012 from Air Force Engineering University. Currently, he is a lecturer in Information and Navigation College, Air Force Engineering University. His research interests include mission planning technique and military organizational analysis. E-mail: dumu3110728@126.com

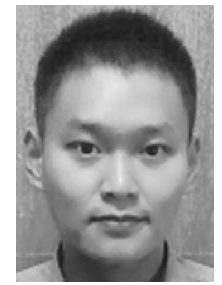

ZHONG Yun was born in 1990. He received his B.S. degree in network engineering from Air Force Engineering University in 2012, his M.S. degree in information and communication engineering from Air Force Engineering University in 2015. He is currently a Ph.D. candidate of Air Force Engineering University. His research interests include command information system, and mission planning.

E-mail: pandawlj@126.com

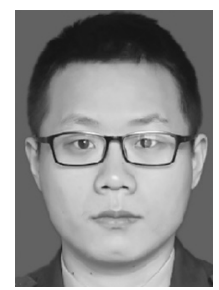

WANG Xun was born in 1990. He received his B.S. degree in communication engineering from Shandong University in 2013, and his M.S. degree in command information system from Air Force Engineering University in 2016. He is currently a Ph.D. candidate of Air Force Engineering University. His research interests include command information system and mission planning.

E-mail: wxkgdxy@163.com 